\title{
Health Care Resource Utilization Following Initiation of a Triptan: A Retrospective Claims Analysis
}

\author{
Andrew Messali, PharmD; Gary Owens, MD; Lisa Bloudek, PharmD, MS; \\ Shashidhar Kori, MD; Ashley Cole, MPH; and Jenny Chia, PhD
}

\begin{abstract}
BACKGROUND: 23 million Americans suffer from migraine headaches, incurring more than $\$ 1$ billion in direct medical costs each year (with another $\$ 13$ billion in indirect productivity losses). Triptans are the most common treatment of choice for these patients; however, adherence and persistence to triptans are poor. Partly due to poor adherence to therapy, the ability of triptans to reduce the utilization of other medical services and prescription drugs remains unclear.
\end{abstract}

OBJECTIVES: To (a) assess changes in the utilization of medical services and relevant prescription drugs after patients suffering from episodic migraines begin triptan therapy and (b) further investigate the relationship between concomitant opioid use among triptan-treated migraine patients and further utilization of medical services and prescription drugs.

METHODS: A retrospective analysis of pharmacy and medical insurance claims was carried out using a large and nationally representative database. The utilization patterns of episodic migraine patients were observed for 12 to 24 months prior to their first triptan prescription and 12 to 24 months following that prescription. Resource utilization included physician office visits, diagnostic imaging, emergency room use, inpatient hospitalization, opioid prescriptions, migraine prophylaxis prescriptions, and acetaminophen or nonsteroidal anti-inflammatory prescriptions. Results were stratified according to triptan-switching behavior.

RESULTS: The analytic sample included 9,521 migraine patients who were followed for a median of $\mathbf{5 5 0}$ days before and after their first triptan fill. $40.9 \%$ of these patients filled their triptan prescriptions only once (index fill). Another $40.3 \%$ filled a triptan prescription at least twice and never switched their triptan brand. $15.6 \%$ of patients switched their triptan prescriptions once, and $3.2 \%$ of patients switched their triptan prescriptions twice or more. The only group to display significant reductions in resource utilization following the prescription of a triptan was the cohort that never refilled the medication, potentially suggestive of misdiagnosis. Either no significant change or a significant increase in resource utilization was seen in all other cases. The ability of triptans to reduce resource utilization seemed to be lower among patients who switched triptans more often. Patients that concomitantly used opioid medications in addition to triptans also used significantly more resources than migraine patients who were not treated with opioids.

CONCLUSION: Contrary to the findings of some previous research, the initiation of triptan therapy did not significantly reduce the utilization of migraine-related medical services or other relevant prescription drugs in this retrospective claims analysis. This may have been due to higher and more realistic rates of triptan switching and discontinuation. Consistent with previous findings, patients using concomitant opioids used more migraine-related health care resources.

J Manag Care Pharm. 2014;20(4):368-75

Copyright $\odot 2014$, Academy of Managed Care Pharmacy. All rights reserved.

\section{What is already known about this subject}

- Triptans are the most common option for the acute treatment of migraines

- Studies have shown that adherence and persistence to triptan therapy are poor.

- The effect of triptan initiation on migraine-related health care resource utilization remains unclear.

\section{What this study adds}

- In this study, we retrospectively observe the utilization patterns of episodic migraine patients before and after beginning triptan therapy.

- Triptan initiation was not associated with significant reductions in the utilization of any medical services or relevant prescription drugs.

$\mathrm{M}$ igraine headaches are among the most common neurological disorders in the United States and other Western countries. Eighteen percent of women and $6 \%$ of men in the United States above the age of 12 suffer from migraines-roughly 23 million Americans. ${ }^{1}$ Migraine prevalence is highest among 35 to 45 year olds, Caucasian women, and lower-income households. ${ }^{2}$ These factors associated with migraine prevalence have been consistently described in research conducted in the United States, Canada, and several European countries. ${ }^{3-5}$

The direct costs of migraine treatment have been estimated to exceed $\$ 1$ billion each year. ${ }^{1,6}$ About $60 \%$ of these direct costs are attributable to physician office visits, and another $30 \%$ can be attributed to prescription drug treatment. The remaining $10 \%$ consists of inpatient treatment and emergency room visits. As is the case with many chronic diseases, the indirect costs of migraine headaches far outweigh the direct costs. The attributable productivity losses to American employers have been estimated to be roughly $\$ 13$ billion annually. The majority of these productivity losses ( $\$ 8$ billion each year) are the result of migraine-related missed workdays.

From a payer's perspective, an important aspect of the value of prescription medications lies in their potential to prevent more costly utilization of health care resources, such as emergency room visits and inpatient hospitalizations, by managing 
disease progression or alleviating symptoms. ${ }^{7}$ For example, it has been estimated that improving adherence to antipsychotic medications could reduce acute care admissions by $12.3 \%$ and inpatient treatment days by $13.1 \%$, resulting in $\$ 105$ billion in annual savings for Medicaid programs across the United States. ${ }^{8}$ Of course, prescriptions medications can only have this effect if patients remain adherent to therapy.

Triptans are generally the acute treatment of choice for patients suffering from episodic migraines. Because triptans are used as needed to treat migraines when they occur, standard measures of medication adherence, such as medication possession ratio, are not applicable. However, a significant amount of literature describing alternative measures of triptan adherence is available. This literature generally describes the rates of triptan brand switching, one-time filling, and persistency. ${ }^{9,10}$ Persistency rates over 2 years of follow-up have ranged from $46.2 \%$ to $53.2 \%{ }^{9,11}$ The proportion of patients that fill only 1 triptan prescription without any further refills has ranged from $38 \%$ to $56 \%{ }^{9,10,12-15}$ Triptan brand switching is also a common occurrence. Roughly $15 \%$ of triptan users will try a new triptan over 1 year of follow-up. ${ }^{9,10}$ Patients have reported lack of efficacy or side effects as the most common reasons for nonadherence. ${ }^{14}$

In addition to the challenges of adherence and persistence, opioid use among migraine patients has become an increasingly controversial issue. Opioids are generally considered to be a third-line option for treating migraine pain, after simple analgesics and migraine-specific medications. ${ }^{16}$ Recent studies have shown the use of opioids for episodic migraine treatment to be associated with a risk of medication overuse headaches and new-onset chronic migraine. ${ }^{17,18}$ Evidence also suggests that migraine patients treated with opioids generally use more health care resources than other migraine patients. ${ }^{19}$

Therefore, the primary objective of this research was to assess changes in the utilization of medical services and relevant prescription drugs after patients suffering from episodic migraines began triptan therapy. We also sought to further investigate, as a secondary analysis, the relationship between concomitant opioid use among triptan-treated migraine patients and further utilization of medical services and prescription drugs.

\section{Methods}

The Truven Health Analytics (formerly Thomson Reuters Healthcare) MarketScan Database, a large and nationally representative database of medical and pharmacy claims, was used to conduct the analysis. With commercial claims data spanning the period 2006 to 2010, we began by identifying patients diagnosed with episodic migraine (ICD-9-CM [International Classification of Diseases, Ninth Revision, Clinical Modification] 346.0x, 346.1x, 346.2x) who had at least 1 fill for a triptan medication. Patients whose first observed migraine diagno- sis in the data came on or after the date of their first triptan fill were excluded. The date of first triptan fill became each patient's index date. The pre-index (or pre-triptan) period was defined as the time from first observed diagnosis of migraine to first fill of a triptan medication. The length of this time varied from patient to patient. The post-index (or post-triptan) period, which began immediately after the first triptan fill, was defined as a length of time equal to the pre-index period. Patients who were not continuously enrolled through the pre- and postindex periods were excluded. Finally, any patients who had a pre-index period of less than 1 year were also excluded to ensure adequate observation time (Figure 1). Three sensitivity analyses requiring only 1,3 , and 6 months of pre-index time were also carried out.

Throughout this analysis, triptan medications included oral sumatriptan, rizatriptan, naratriptan, frozatriptan, almotriptan, eletriptan, and zolmitriptan. Health care resources included physician office visits, emergency room visits, inpatient hospitalizations, diagnostic imaging, opioid prescription fills, migraine prophylaxis medication fills, and fills for acetaminophen or nonsteroidal anti-inflammatory drugs (NSAIDs). All claims for physician office visits, emergency room visits, inpatient hospitalizations, and diagnostic imaging were required to have an associated diagnosis of migraine (ICD-9-CM 346.xx). Diagnostic imaging procedures included all CT and MRI scans to the head. Migraine prophylaxis prescriptions included all medications that have received a "strong" evidence rating for their abilities to prevent episodic migraine attacks from the American Academy of Neurology (AAN), which include propranolol, timolol, metoprolol, topiramate, divalproex, and valproate.

To assess the impact of switching between triptan brands, the primary results were stratified according to the number of unique generic triptans that patients filled during their postindex period. Those patients who filled their triptan prescriptions only once (the index fill) were considered as a separate stratum.

As a secondary analysis, the health care resource utilization of those patients within the analytic sample who used opioid medications during their post-index period was compared with that of those patients who did not use opioid medications. For the purposes of this analysis, health care resource utilization included all of the services and medications previously defined, except for opioid prescriptions. In order to exclude patients temporarily using opioids for reasons unrelated to the treatment of migraine pain, 2 fills of an opioid medication were required to be considered a concomitant opioid user.

Because the frequency distributions of health care resource utilization are often highly skewed, nonparametric methods were used to compare the frequency of utilization before and after triptan initiation (primary analysis) and between opioid users and nonusers (secondary analysis). Because the 


\section{FIGURE 1 Sample Selection Process}

Patients with episodic migraine diagnosis (ICD-9-CM 346.0X, 346.1X, 346.2X), MarketScan Commercial, 2006-2011 $(\mathrm{N}=787,912)$

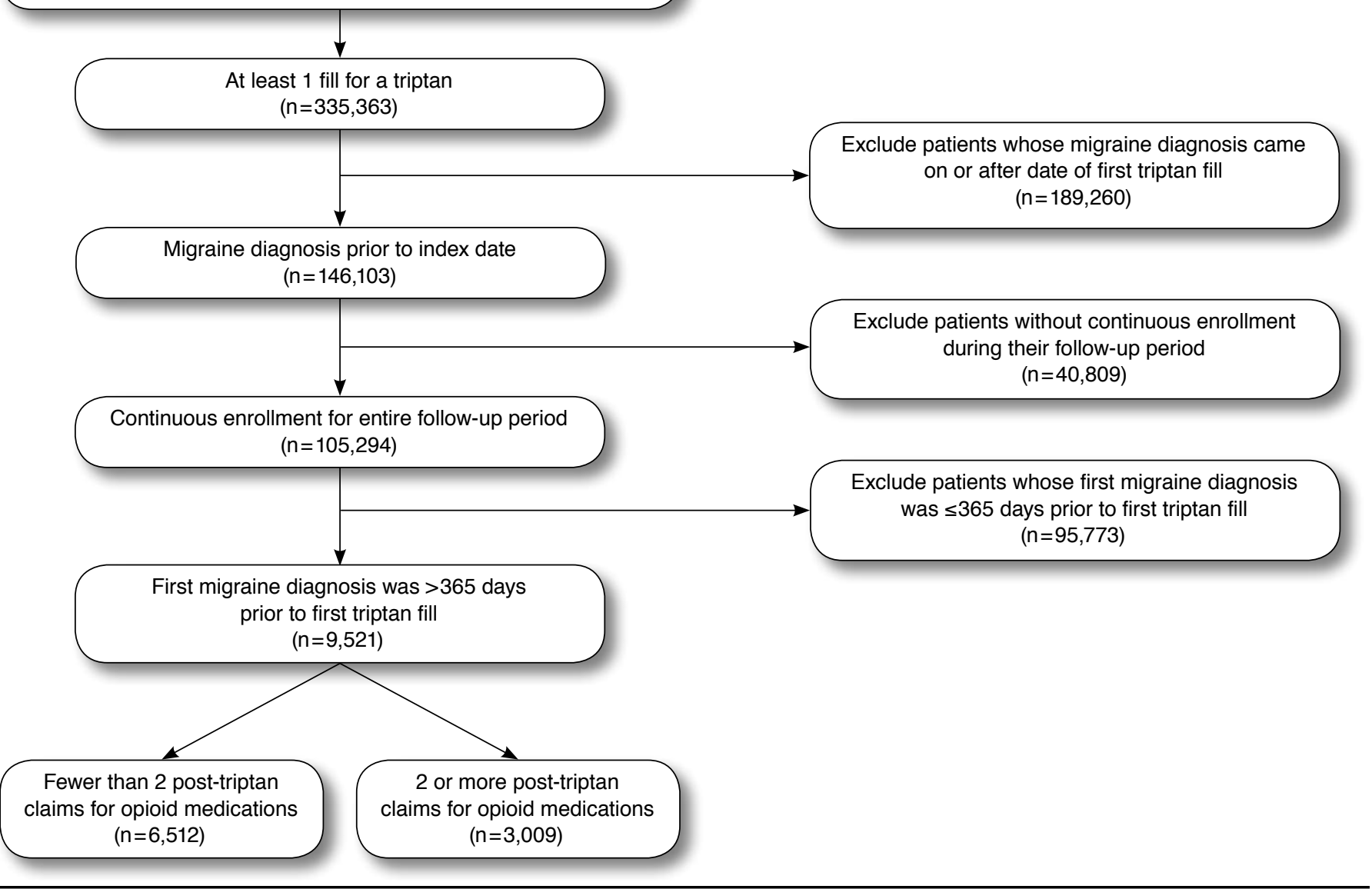

ICD-9-CM = International Classification of Diseases, Ninth Revision, Clinical Modification.

observations in the primary analysis are paired, signed rank tests were used. Because the observations in the secondary analysis were not paired, Wilcoxon rank-sum tests were used.

\section{Results}

The final analytic sample included 9,521 patients, each of whom had between 12 and 24 months of pre-index enrollment and 12 and 24 months of post-index enrollment. The median follow-up time was 550 days pre-triptan and 550 days post-triptan. Among these patients, 3,891 (40.9\%) filled their triptan prescriptions only once (index fill). Another 3,840 (40.3\%) filled their triptan prescriptions at least twice and never switched their triptan brand during follow-up; 1,487 (15.6\%) filled their triptan prescriptions at least twice and switched their triptan brand once during follow-up; and 303 (3.2\%) filled their triptan prescriptions at least twice and switched their triptan brand twice or more during follow-up (Figure 2). The age, gender, and length of follow-up of these triptan-switching strata are described in Table 1.

Among patients who filled their triptan prescriptions only once, significant reductions in the rates of physician office visits $(P<0.01)$ and diagnostic imaging $(P<0.01)$ were seen following triptan initiation. No significant changes were seen in the rates of emergency room visits or inpatient hospitalizations. Among patients who filled their triptan prescriptions at least twice but never switched their triptan brand, a significant increase in the rate of physician office visits was seen following triptan initiation $(P<0.01)$, with no significant changes in the rates of utilizing other medical services. Among patients who filled their triptan prescriptions at least twice and switched their triptan brand once during follow-up, significant increases 
FIGURE 2 Triptan Brand-Switching Behavior Among the Complete Patient Sample

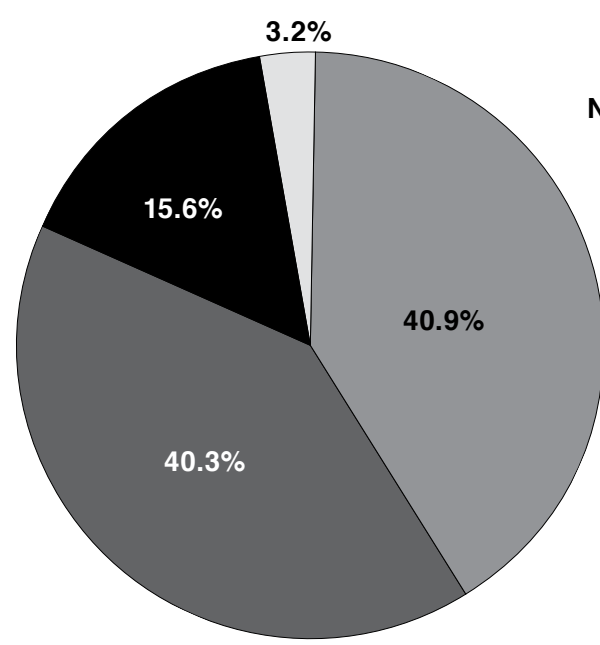

0 Refills

1 Triptan

2 Triptans

$3+$ Triptans

Note: Patients categorized as using 1 triptan, 2 triptans, or 3+ triptans filled their triptan prescriptions at least twice.

in the rates of physician office visits $(P<0.01)$, emergency room visits $(P<0.01)$, and inpatient hospitalization $(P<0.01)$ occurred after triptan initiation. No significant change in the rate of diagnostic imaging procedures was seen. Finally, among patients who filled their triptan prescriptions at least twice and switched their triptan brand twice or more, we saw significant increases in the rates of physician office visits $(P<0.01)$ and emergency room visits $(P<0.01)$, with no significant changes in the rates of diagnostic imaging or inpatient hospitalization (Table 2).

No significant change was seen in the rate of filling migraine prophylaxis medications among patients who never refilled their triptan prescriptions. Aside from this single exception, the rates of filling opioid medications, migraine prophylaxis medi- cations, and acetaminophen/NSAID medications increased after triptan initiation, regardless of triptan-switching behavior during follow-up (all $P<0.01$; Table 2 ). The sensitivity analyses requiring only 1,3 , and 6 months of pre-index observation did not yield any substantially different results.

Using the classification of opioid users and nonusers previously described, our analytic sample of 9,521 patients was divided into 3,009 concomitant opioid users and 6,512 nonusers. The age, gender, and follow-up time of concomitant opioid users and nonusers are described in Table 1. The rates of all medical services and filling of relevant prescription medications were significantly higher among opioid users, compared with nonusers (Table 3). Again, the sensitivity analyses requiring only 1,3 , and 6 months of pre-index observation produced similar results.

\section{Discussion}

Our results indicate that initiating triptan therapy does not significantly reduce the utilization of other migraine-related medical services and prescription medications. In fact, significant increases in several categories of health care resource utilization were seen after patients began using a triptan. Furthermore, the ability of triptans to reduce the utilization of other relevant medical services and prescription drugs appeared to be substantially worse among patients who switched their triptan brand more often during follow-up. Finally, patients who used opioids along with triptans during the post-triptan period did utilize all medical services and prescription drugs at higher rates than patients who did not use opioids.

Because our sample size was quite large, relatively small differences appeared statistically significant. Although we have reported $P$ values, we believe that these results should be considered in the context of clinical significance. Several small but statistically significant increases in resource utilization were seen after patients began triptan therapy. This could be interpreted a number of ways, but what is more important is that triptan initiation did not decrease resource utilization.

TABLE 1 Patient Characteristics and Follow-Up Time Stratified by Triptan-Switching Behavior and Opioid Use

\begin{tabular}{|c|c|c|c|c|c|c|c|c|c|c|c|c|c|c|}
\hline & \multicolumn{2}{|c|}{ All Patients } & \multicolumn{2}{|c|}{0 Refills } & \multicolumn{2}{|c|}{1 Triptan } & \multicolumn{2}{|c|}{2 Triptans } & \multicolumn{2}{|c|}{ 3+ Triptans } & \multicolumn{2}{|c|}{ Opioid Users } & \multicolumn{2}{|c|}{ Nonusers } \\
\hline Follow-up days, median (IQR) & \multicolumn{2}{|c|}{$550(427-718)$} & \multicolumn{2}{|c|}{$512(422-699)$} & \multicolumn{2}{|c|}{$531(428-718)$} & \multicolumn{2}{|c|}{$536(434-730)$} & \multicolumn{2}{|c|}{$574(452-730)$} & \multicolumn{2}{|c|}{$532(427-711)$} & \multicolumn{2}{|c|}{$520(428-729)$} \\
\hline Female, n (\%) & 7,983 & $(83.85)$ & 3,246 & $(83.42)$ & 3,186 & $(82.97)$ & 1,287 & $(86.55)$ & 264 & $(87.13)$ & 5,407 & $(83.03)$ & 2,576 & $(85.61)$ \\
\hline \multicolumn{15}{|l|}{ Age, n (\%) } \\
\hline $0-17$ & 871 & $(9.15)$ & 368 & $(9.46)$ & 336 & $(8.75)$ & 140 & $(9.41)$ & 27 & $(8.91)$ & 83 & $(2.76)$ & 788 & $(12.10)$ \\
\hline $18-29$ & 1,388 & $(14.58)$ & 667 & (17.14) & 446 & $(11.61)$ & 222 & (14.93) & 53 & $(17.49)$ & 357 & $(11.86)$ & 1,031 & $(15.83)$ \\
\hline $30-39$ & 2,511 & $(26.37)$ & 1,048 & $(26.93)$ & 927 & $(24.14)$ & 442 & $(29.72)$ & 94 & $(31.02)$ & 881 & $(29.28)$ & 1,630 & $(25.03)$ \\
\hline $40-49$ & 2,654 & (27.88) & 994 & $(25.55)$ & 1,177 & $(30.65)$ & 402 & $(27.03)$ & 81 & $(26.73)$ & 917 & $(30.48)$ & 1,737 & $(26.67)$ \\
\hline $50-59$ & 1,782 & $(18.72)$ & 688 & $(17.68)$ & 814 & $(21.20)$ & 238 & $(16.01)$ & 42 & $(13.86)$ & 664 & $(22.07)$ & 1,118 & (17.17) \\
\hline $60-65$ & 315 & (3.31) & 126 & (3.24) & 140 & $(3.65)$ & 43 & $(2.89)$ & 6 & (1.98) & 107 & $(3.56)$ & 208 & (3.19) \\
\hline
\end{tabular}


Health Care Resource Utilization Following Initiation of a Triptan: A Retrospective Claims Analysis

TABLE 2 Health Care Resource Utilization Before and After Triptan Initiation Stratified by Triptan-Switching Behavior

\begin{tabular}{|c|c|c|c|c|c|c|c|c|}
\hline \multirow[b]{3}{*}{ Office visits } & \multirow{2}{*}{$\begin{array}{c}\text { Triptan } \\
\text { Category }\end{array}$} & \multirow{2}{*}{$\mathrm{N}$} & \multicolumn{3}{|c|}{ Pre-triptan Utilization } & \multicolumn{2}{|c|}{ Post-triptan Utilization } & \multirow[b]{2}{*}{$P$ Value $^{\mathrm{a}}$} \\
\hline & & & Mean (SD) & \multicolumn{2}{|c|}{ Median (IQR) } & Mean (SD) & \multirow{2}{*}{$\frac{\text { Median (IQR) }}{1(0-3)}$} & \\
\hline & 0 Refills & 3,891 & $2.52 \quad(7.17)$ & 1 & $(0-3)$ & $2.30 \quad(6.98)$ & & $<0.01$ \\
\hline & 1 Triptan & 3,840 & $2.54 \quad(4.41)$ & 1 & $(0-3)$ & $2.76 \quad(4.66)$ & $2(0-3)$ & $<0.01$ \\
\hline & 2 Triptans & 1,487 & $3.08 \quad(5.17)$ & 2 & $(1-4)$ & $4.45 \quad(6.05)$ & $3(1-6)$ & $<0.01$ \\
\hline & $3+$ Triptans & 303 & $3.78 \quad(4.87)$ & 2 & $(1-5)$ & $7.46 \quad(8.65)$ & $5(3-10)$ & $<0.01$ \\
\hline \multirow{4}{*}{$\begin{array}{l}\text { Diagnostic } \\
\text { imaging }\end{array}$} & 0 Refills & 3,891 & $0.06 \quad(0.28)$ & 0 & $(0-0)$ & $0.04 \quad(0.24)$ & $0(0-0)$ & $<0.01$ \\
\hline & 1 Triptan & 3,840 & $0.05 \quad(0.23)$ & 0 & $(0-0)$ & $0.04 \quad(0.22)$ & $0(0-0)$ & 0.46 \\
\hline & 2 Triptans & 1,487 & $0.07 \quad(0.29)$ & 0 & $(0-0)$ & $0.09 \quad(0.32)$ & $0(0-0)$ & 0.08 \\
\hline & $3+$ Triptans & 303 & $0.10 \quad(0.33)$ & 0 & $(0-0)$ & $0.17 \quad(0.45)$ & $0(0-0)$ & 0.06 \\
\hline \multirow{4}{*}{$\begin{array}{l}\text { Emergency } \\
\text { room }\end{array}$} & 0 Refills & 3,891 & $0.19 \quad(1.15)$ & 0 & $(0-0)$ & $0.18 \quad(1.07)$ & $0(0-0)$ & 0.17 \\
\hline & 1 Triptan & 3,840 & $0.19 \quad(0.96)$ & 0 & $(0-0)$ & $0.18 \quad(0.85)$ & $0(0-0)$ & 0.81 \\
\hline & 2 Triptans & 1,487 & $0.31 \quad(1.28)$ & 0 & $(0-0)$ & $0.40 \quad(1.55)$ & $0(0-0)$ & $<0.01$ \\
\hline & 3+ Triptans & 303 & $0.34 \quad(1.04)$ & 0 & $(0-0)$ & $0.62 \quad(1.50)$ & $0(0-0)$ & $<0.01$ \\
\hline \multirow[t]{4}{*}{ Hospitalization } & 0 Refills & 3,891 & $0.03 \quad(0.20)$ & 0 & $(0-0)$ & $0.03 \quad(0.21)$ & $0(0-0)$ & 0.43 \\
\hline & 1 Triptan & 3,840 & $0.04 \quad(0.29)$ & 0 & $(0-0)$ & $0.03 \quad(0.22)$ & $0(0-0)$ & 0.06 \\
\hline & 2 Triptans & 1,487 & $0.04 \quad(0.24)$ & 0 & $(0-0)$ & $0.08 \quad(0.40)$ & $0(0-0)$ & $<0.01$ \\
\hline & $3+$ Triptans & 303 & $0.10 \quad(0.37)$ & 0 & $(0-0)$ & $0.14 \quad(0.46)$ & $0(0-0)$ & 0.17 \\
\hline \multirow[t]{4}{*}{ Opioids } & 0 Refills & 3,891 & $3.31 \quad(7.99)$ & 1 & $(0-2)$ & $3.72 \quad(8.55)$ & $1(0-3)$ & $<0.01$ \\
\hline & 1 Triptan & 3,840 & $3.31 \quad(7.61)$ & 1 & $(0-3)$ & $3.86 \quad(8.54)$ & $1(0-3)$ & $<0.01$ \\
\hline & 2 Triptans & 1,487 & $\begin{array}{ll}4.26 \quad(9.38) \\
\end{array}$ & 1 & $(0-4)$ & $5.17(10.31)$ & $1(0-5)$ & $<0.01$ \\
\hline & 3+ Triptans & 303 & 5.57 (11.62) & 1 & $(0-6)$ & $7.04(12.95)$ & $2(0-8)$ & $<0.01$ \\
\hline \multirow{4}{*}{$\begin{array}{l}\text { Acetaminophen/ } \\
\text { NSAIDs }\end{array}$} & 0 Refills & 3,891 & $4.13 \quad(8.37)$ & 1 & $(0-4)$ & $4.40 \quad(8.65)$ & $1(0-4)$ & $<0.01$ \\
\hline & 1 Triptan & 3,840 & $4.02 \quad(7.54)$ & 1 & $(0-4)$ & $4.72 \quad(8.55)$ & $2(0-5)$ & $<0.01$ \\
\hline & 2 Triptans & 1,487 & $5.21 \quad(9.64)$ & 2 & $(0-5)$ & $6.51 \quad(10.95)$ & $2(1-7)$ & $<0.01$ \\
\hline & $3+$ Triptans & 303 & $7.03(10.63)$ & 3 & $(1-9)$ & $9.36(12.66)$ & $5(1-13)$ & $<0.01$ \\
\hline \multirow{4}{*}{$\begin{array}{l}\text { Migraine prophylaxis } \\
\text { medications }\end{array}$} & 0 Refills & 3,891 & $1.94 \quad(4.44)$ & 0 & $(0-1)$ & $2.04 \quad(4.46)$ & $0(0-2)$ & 0.06 \\
\hline & 1 Triptan & 3,840 & $2.47 \quad(5.01)$ & 0 & $(0-2)$ & $3.18 \quad(5.58)$ & $0(0-5)$ & $<0.01$ \\
\hline & 2 Triptans & 1,487 & $2.43 \quad(4.95)$ & 0 & $(0-2)$ & $3.79 \quad(5.79)$ & $1(0-6)$ & $<0.01$ \\
\hline & $3+$ Triptans & 303 & $2.68 \quad(4.32)$ & 0 & $(0-4)$ & $5.81 \quad(6.59)$ & $4(0-10)$ & $<0.01$ \\
\hline
\end{tabular}

aP value for signed rank test. Patients categorized as using 1 triptan, 2 triptans, or $3+$ triptans filled their triptan prescriptions at least twice.

$I Q R=$ interquartile range; NSAIDs = nonsteroidal anti-inflammatory drugs; $S D=$ standard deviation.

The rates of 1-time triptan filling and triptan brand switching that we observed were consistent with previous literature. ${ }^{9-15}$ The fact that patients who filled their triptan prescriptions only once showed a significant decrease in the rates of physician office visits and diagnostic imaging after their index triptan fill is potentially suggestive of misdiagnosis. If these patients truly suffered from migraines, one would expect their medical services utilization rates to continue increasing on a trajectory similar to or worse than that of patients who frequently switched their triptans The fact that triptans seemed less effective at reducing the utilization of other services and medications among patient who switched triptans frequently is logical if triptan switching is viewed as an indicator of ineffectiveness. This view is supported by research indicating that ineffectiveness and side effects are the primary reasons for triptan nonadherence. ${ }^{14}$

Even if patients were receiving large quantities per fill, the rates of migraine prophylaxis medication usage are quite low.
This may simply be due to the fact that we only considered medications that have received a "strong" evidence rating from AAN when counting fills for migraine prophylaxis medications. We have excluded several drugs that have received lower evidence ratings. Because of increased awareness of medication overuse headaches from acute medications, episodic migraine prophylaxis has become an important issue. Despite our relatively strict criteria, it is likely that these medications are being underutilized.

Previous studies of the potential cost savings associated with triptan use have been limited to small, open-label prospective studies following patients treated with sumatriptan. Three such studies, following between 100 and 200 patients for 6 to 18 months before and after sumatriptan initiation, have concluded that sumatriptan therapy significantly reduced the utilization of medical services and/or prescription drugs. ${ }^{20-22}$ Unfortunately, none of these studies reported any information regarding patient adherence to therapy. It is possible that 
Health Care Resource Utilization Following Initiation of a Triptan: A Retrospective Claims Analysis

\section{TABLE 3 Health Care Resource Utilization After Triptan Initiation} Among Concomitant Opioid Users and Nonusers

\begin{tabular}{|c|c|c|c|c|c|c|c|c|c|}
\hline \multirow[b]{3}{*}{ Office visits } & \multicolumn{4}{|c|}{$\begin{array}{l}\text { Opioid Users } \\
(\mathrm{N}=3,009)\end{array}$} & \multicolumn{4}{|c|}{$\begin{array}{l}\text { Opioid Nonusers } \\
\qquad(\mathrm{N}=6,512)\end{array}$} & \multirow{3}{*}{$\frac{P \text { Value }^{\mathrm{a}}}{<0.01}$} \\
\hline & \multicolumn{2}{|c|}{ Mean (SD) } & \multicolumn{2}{|c|}{ Median (IQR) } & \multicolumn{2}{|c|}{ Mean (SD) } & \multicolumn{2}{|c|}{ Median (IQR) } & \\
\hline & 3.13 & $(6.25)$ & 2 & $(0-4)$ & 1.77 & $(2.88)$ & 1 & $(0-2)$ & \\
\hline Diagnostic imaging & 0.06 & $(0.28)$ & 0 & $(0-0)$ & 0.04 & $(0.20)$ & 0 & $(0-0)$ & $<0.01$ \\
\hline Emergency room & 0.35 & $(1.38)$ & 0 & $(0-0)$ & 0.08 & $(0.45)$ & 0 & $(0-0)$ & $<0.01$ \\
\hline Hospitalization & 0.06 & $(0.30)$ & 0 & $(0-0)$ & 0.02 & $(0.15)$ & 0 & $(0-0)$ & $<0.01$ \\
\hline Acetaminophen/NSAIDs & 2.19 & $(4.05)$ & 1 & $(0-3)$ & 0.93 & $(2.19)$ & 0 & $(0-1)$ & $<0.01$ \\
\hline Migraine prophylaxis medications & 2.61 & $(3.99)$ & 0 & $(0-4)$ & 1.77 & $(3.45)$ & 0 & $(0-2)$ & $<0.01$ \\
\hline
\end{tabular}

a $P$ value for rank-sum test.

$I Q R=$ interquartile range; NSAIDs = nonsteroidal anti-inflammatory drugs; SD = standard deviation.

patients volunteering to participate in these open-label studies will display better adherence, compared to patients not participating in research.

A retrospective analysis of medical and pharmacy claims from 2 health maintenance organizations found a significant increase in emergency room visits and physician office visits following the initiation of injectable sumatriptan. ${ }^{23}$ However, this analysis observed only 49 patients who began using injectable sumatriptan soon after it was first available in the United States, so the results might not be generalizable to users of oral triptans. More recently, another retrospective analysis of claims from an employer-sponsored health plan found that, after controlling for several covariates, migraine patients who used antimigraine medications had significantly lower migraine-related resource utilization compared with untreated migraine patients. ${ }^{24}$ However, the analysis grouped together triptans, ergotamines, and NSAIDs as antimigraine medications. Furthermore, the analysis was entirely cross-sectional, and although regression models were used to control for several covariates, differences between treated and untreated migraine patients are likely to persist.

Several reviews of the pharmacoeconomics of triptans have acknowledged some of these mixed results. ${ }^{25,26}$ It seems possible that differences in research methods have produced some study populations that are more adherent to therapy and others that are less so. This adherence may then significantly impact the ability of triptans to control medical and pharmacy utilization. However, a retrospective analysis of a large, nationally representative database of medical and pharmacy claims, such as this one, is considered to be one of the best ways of measuring the impact of triptans in the context of their normal daily use and typical patient adherence.

The observation that patients who use opioids concomitantly with a triptan to manage migraine-related pain tend to use more health care services and prescription medications is consistent with previous literature. ${ }^{19}$ These findings are also logical because patients who receive prescriptions for opioids probably have more severe or more frequent migraines that those who do not receive such prescriptions.

The ability of prescription medications to alleviate the demand for other medical services is only one of many factors that payers may consider. Although we have found that triptans, as they are used in the real world, may not reduce other medical resource utilization, relatively fast reductions in migraine pain and improved quality of life should also be considered.

\section{Limitations}

This analysis is subject to a few limitations, the most important of which concerns our decision to exclude all patients whose first triptan fill occurred within 1 year of their migraine diagnosis. Some length of time between diagnosis and first triptan fill is required to define the pre-period so that a pre/post design could be used. This pre-period also needed to be long enough to observe the utilitization of health care resources, some of which, such as hospitalization, are quite rare. We chose to require 1 year between diagnosis and first triptan fill for 2 reasons: first, to prevent the utilization of medical services associated with diagnosing the migraine disorder itself from being counted in the post-triptan period and, second, because we suspected that there might be a natural tendency for the utilization of medical services to increase over the period immediately following diagnosis, independent of any triptan use. This might occur due to increases in frequency or severity of the migraines or simply because newly diagnosed patients might be more prone to seeking treatment. Therefore, in our view, excluding patients without a full year between diagnosis and their first triptan fill prevented several potential biases towards finding more utilization during the post-triptan period.

This could have also induced a sample selection bias if triptans are more effective when initiated soon after migraine 
diagnosis. This phenomenon is true of some medication classes. However, we are unaware of any literature to support this hypothesis with respect to triptan therapy. A sample selection bias also could have been introduced if patients who wait at least 1 year after receiving a migraine diagnosis to initiate a triptan are less responsive to triptan therapy for any other reasons. However, we are also unaware of any evidence to support this idea. In fact, it seems more likely that patients who do not initiate triptan therapy soon after migraine diagnosis are less recalcitrant, simply because they were able to manage their symptoms adequately, presumably using over-the-counter medications, for a longer period of time. The results of our sensitivity analyses requiring only 1,3 , and 6 months of pre-index enrollment, which were consistent with our primary results, also indicate that our inclusion criteria have not introduced a sample selection bias.

Another limitation involves the potential for episodic migraine to progress into chronic migraine. A patient is considered to have progressed to chronic migraine if the frequency of migraine headaches occurs at least 15 days per month for more than 3 months. ${ }^{27}$ If a significant proportion of the patients in our analysis were experiencing progressively more frequent or more severe migraines, then this trend could bias us towards finding more frequent utilization in the post-triptan period. However, population studies have estimated that only about $2.5 \%$ of episodic migraine patients will progress to chronic migraine over the course of a year. ${ }^{28,29}$ While some of the patients included in this analysis may be progressing over time, it seems unlikely that this would be influencing the utilization patterns of a significant proportion of patients. More modest increases in migraine severity or frequency are also possible but would likely have modest effects on our results.

The secondary analysis of concomitant opioid use was entirely cross-sectional; therefore, we cannot guarantee that initiation of opioid therapy preceded the use of medical services and the filling of other prescription drugs. Finally, in neither the primary analysis nor secondary analysis were we able to observe the use of over-the-counter medications or account for indirect costs such as workplace productivity.

\section{Conclusion}

Contrary to the findings of some previous research, the initiation of triptan therapy did not significantly reduce the utilization of migraine-related medical services or other relevant prescription drugs in this retrospective claims analysis. This may have been due to higher and more realistic rates of triptan switching and discontinuation. Consistent with previous findings, patients using concomitant opioids used more migrainerelated health care resources.

\section{Authors}

ANDREW MESSALI, PharmD, is a Doctoral Student, University of Southern California, Los Angeles, California, and Graduate Intern, Allergan, Inc., Irvine, California; GARY OWENS, MD, is President, Gary Owens Associates, Glen Mills, Pennsylvania; SHASHIDHAR KORI, MD, is Vice President, Clinical Development and Medical Affairs, MAP Pharmaceuticals, Mountain View, California; and LISA BLOUDEK, PharmD, MS, is Manager, Xcenda LLC, Palm Harbor, Florida. ASHLEY COLE, MPH, is Statistical Analyst, and JENNY CHIA, PhD, is Manager/Principal Statistical Analyst, Allergan, Inc., Irvine, California.

AUTHOR CORRESPONDENCE: Andrew Messali, PharmD, Allergan, Inc., 2525 Dupont Dr., Irvine, CA 92612-1959.

E-mail: Messali_Andrew@Allergan.com.

\section{DISCLOSURES}

This study was funded by Allergan, Inc. The sponsor and all authors directed the research design and reviewed all major research decisions (i.e., study instruments, sampling, and analyses). The authors had full access to all data and had final responsibility for the decision to submit for publication.

Messali served as an intern at Allergen, Inc., when this study was conducted. Owens serves as a consultant and as a member of the advisory boards of Amgen, Allergan, Eli Lilly, Boston Scientific, CardioDx, Novocure, Genzyme, Genentech/Roche, Johnson \& Johnson, and Medimmune.

Messali and Bloudek participated in planning the study and interpreting the results. Cole and Chia participated in collecting the data and doing the analysis. Messali wrote the initial first draft of the article. All authors were involved in interpreting the results, planning the article, critical review, and editing of the first draft and subsequent revisions of the paper. All authors approved the manuscript before submission.

\section{REFERENCES}

1. Lipton RB, Bigal ME. Ten lessons on the epidemiology of migraine. Headache. 2007;(47 Suppl 1):S2-S9.

2. Lipton RB, Stewart WF, Diamond S, Diamond ML, Reed M. Prevalence and burden of migraine in the United States: data from the American Migraine Study II. Headache. 2001;41(7):646-57.

3. Gobel H, Petersen-Braun M, Soyka D. The epidemiology of headache in Germany: a nationwide survey of a representative sample on the basis of the headache classification of the International Headache Society. Cephalalgia. 1994;14(2):97-106.

4. Henry P, Michel P, Brochet B, Dartigues JF, Tison S, Salamon R. A nationwide survey of migraine in France: prevalence and clinical features in adults. GRIM. Cephalalgia. 1992;12(4):229-37; discussion 186.

5. Pryse-Phillips W, Findlay H, Tugwell P, Edmeads J, Murray TJ, Nelson RF. A Canadian population survey on the clinical, epidemiologic, and societal impact of migraine and tension-type headache. Can J Neurol Sci. 1992;19(3):333-39.

6. Hu XH, Markson LE, Lipton RB, Stewart WF, Berger ML. Burden of migraine in the United States: disability and economic costs. Arch Intern Med. 1999;159(8):813-18.

7. Kleinke JD. The price of progress: prescription drugs in the health care market. Health Aff (Millwood). 2001;20(5):43-60.

8. Marcus SC, Olfson M. Outpatient antipsychotic treatment and inpatient costs of schizophrenia. Schizophr Bull. 2008;34(1):173-80. 
9. Panconesi A, Pavone E, Franchini M, et al. Triptans: low utilization and high turnover in the general population. Cephalalgia. 2010;30(5):576-81.

10. Ifergane G, Wirguin I, Shvartzman P. Triptans-why once? Headache. 2006;46(8):1261-63.

11. Katic BJ, Krause SJ, Tepper SJ, Hu HX, Bigal ME. Adherence to acute migraine medication: what does it mean, why does it matter? Headache. 2010;50(1):117-29.

12. Etemad LR, Yang W, Globe D, Barlev A, Johnson KA. Costs and utilization of triptan users who receive drug prophylaxis for migraine versus triptan users who do not receive drug prophylaxis. J Manag Care Pharm. 2005;11(2):137-44. Available at: http://amcp.org/WorkArea/DownloadAsset. aspx?id=7121.

13. Lugardon S, Roussel H, Sciortino V, Montastruc JL, Lapeyre-Mestre M. Triptan use and risk of cardiovascular events: a nested-case-control study from the French health system database. Eur J Clin Pharmacol. 2007;63(8):801-07.

14. Rahimtoola H, Buurma H, Tijssen CC, Leufkens HG, Egberts AC. Single use of sumatriptan: a patient interview study. Headache. 2003;43(2):109-16.

15. Gaist D, Hallas J, Sindrup SH, Gram LF. Is overuse of sumatriptan a problem? A population-based study. Eur J Clin Pharmacol. 1996;50(3):161-65.

16. Silberstein SD. Practice parameter: evidence-based guidelines for migraine headache (an evidence-based review): report of the Quality Standards Subcommittee of the American Academy of Neurology. Neurology. 2000;55(6):754-62

17. Radat F, Lanteri-Minet M. What is the role of dependence-related behavior in medication-overuse headache? Headache. 2010;50(10):1597-611.

18. Bigal ME, Lipton RB. Excessive opioid use and the development of chronic migraine. Pain. 2009;142(3):179-82.
19. Buse DC, Pearlman SH, Reed ML, Serrano D, Ng-Mak DS, Lipton RB. Opioid use and dependence among persons with migraine: results of the AMPP study. Headache. 2012;52(1):18-36.

20. Litaker DG, Solomon GD, Genzen JR. Impact of sumatriptan on clinic utilization and costs of care in migraineurs. Headache. 1996;36(9):538-41.

21. Lofland JH, Johnson NE, Batenhorst AS, Nash DB. Changes in resource use and outcomes for patients with migraine treated with sumatriptan: a managed care perspective. Arch Intern Med. 1999;159(8):857-63.

22. Cohen JA, Beall D, Beck A, et al. Sumatriptan treatment for migraine in a health maintenance organization: economic, humanistic, and clinical outcomes. Clin Ther. 1999;21(1):190-204.

23. Streator SE. Pharmacoeconomic impact of injectable sumatriptan on migraine-associated health care costs. Am J Manag Care. 1996;2(2):139-43.

24. Wu J, Hughes MD, Hudson MF, Wagner PJ. Antimigraine medication use and associated health care costs in employed patients. J Headache Pain. 2012;13(2):121-27.

25. Coukell AJ, Lamb HM. Sumatriptan. A pharmacoeconomic review of its use in migraine. Pharmacoeconomics. 1997;11(5):473-90.

26. Caro JJ, Getsios D. Pharmacoeconomic evidence and considerations for triptan treatment of migraine. Expert Opin Pharmacother. 2002;3(3):237-48.

27. Lipton RB, Bigal ME, Steiner TJ, Silberstein SD, Olesen J. Classification of primary headaches. Neurology. 2004;63(3):427-35.

28. Bigal ME, Lipton RB. Modifiable risk factors for migraine progression (or for chronic daily headaches)_clinical lessons. Headache. 2006;(46 Suppl 3): S144-46.

29. Lipton RB. Tracing transformation: chronic migraine classification, progression, and epidemiology. Neurology. 2009;72(5 Suppl):S3-S7. 